\title{
A MAC Scheme with QoS Guarantee for MANETs
}

\author{
Yanbin YANG ${ }^{1,2}$, Yulin WEI ${ }^{2}$ \\ ${ }^{1}$ School of Electronic and Information Engineering, South China University of Technology, Guangzhou, China \\ ${ }^{2}$ Department of Communications, Guangdong Vocational Technology College of Posts \& Telecom, Guangzhou, China \\ Email:sinhei@163.com,TX06wls@126.com \\ Received July 30, 2009; revised August 31, 2009; accepted September 25, 2009
}

\begin{abstract}
IEEE 802.11 distributed coordination function (DCF) can alleviate the collision and hidden station problem, but it doesn't differentiate traffic categories (TC). Therefore, it can't provide sufficient QoS support for different traffic categories. Recently, a new contention-based enhanced distributed channel access (EDCA) scheme was proposed which provides a probabilistic QoS support. In this paper, an adaptive EDCA scheme with QoS guarantee for mobile ad hoc networks (MANETs) is proposed. In this scheme, the EDCA scheme and the token bucket algorithm (TBA) are combined to adjust the contention window (CW). Our scheme provides the traffic differentiation.
\end{abstract}

Keywords: Mobile Ad Hoc Network, Medium Access Control, Distributed Coordination Function, Enhanced Distributed Channel Access, Contention Window

\section{Introduction}

Mobile ad hoc network (MANET) becomes popular because of its low cost and easy deployment. The medium access control (MAC) protocol for this wireless communication system employs a mandatory contention-based channel access function called Distributed Coordination Function (DCF) [1], which is based on the Carrier Sense Multiple Access (CSMA) mechanism. Mobile stations deliver MAC Service Data Units (MSDUs) after detecting that there is no other transmission on the same wireless medium. However, if two stations detect that the channel is free at the same time, a collision occurs. The IEEE 802.11 standard defines a Collision Avoidance (CA) mechanism to reduce the probability of such collisions. As a part of CA, before starting a transmission a station performs a backoff operation. It has to keep sensing the channel for an additional random time after detecting the channel as being idle for a minimum duration called DCF Interframe Space (DIFS). Only if the channel remains idle for this additional random time period, the station is allowed to initiate the transmission. The duration of this random time is determined as a multiple of a slot. Each station maintains a so-called Contention Window $(\mathrm{CW})$, which is used to determine the number of slots a station has to wait before transmission.

To avoid the hidden station problem inherent in CSMA, IEEE 802.11 defines a Request to Send/Clear to
Send (RTS/CTS) scheme, which can be used optionally. Before transmitting data frames, a station has the option to transmit a short RTS frame, followed by the CTS transmission by the receiving station. The RTS and CTS frames include the information of how long it does take to transmit the next data frame, i.e., the first fragment, and the corresponding ACK response. Thus, other stations close to the transmitting station and hidden stations close to the receiving station will not start any transmissions; their timer called Network Allocation Vector (NAV) is set. RTS/CTS helps to protect long data frames against hidden stations. With fragmentation, multiple ACKs are transmitted, whereas with RTS/CTS the MSDU can be efficiently transmitted in a single data frame. Between two consecutive frames in the sequence of RTS, CTS, data, and ACK frames, a Short Interframe Space (SIFS) offers transceivers time to turn around. Because RTS/CTS handshake may introduce some delay especially in case of wireless networks, [2] proposed a method to improve overall throughput of the network where selected RTS packets are dropped based on a node sequence number.

802.11 DCF reduces collision and hidden terminal problem, but it doesn't differentiate TC. Therefore it can't provide sufficient QoS support for different traffic categories. Recently, a new contention-based EDCA scheme was proposed which provides a probabilistic QoS support. [3] presented a new protocol, called dis- 
tributed end-to-end allocation of time slots for real-time traffic (DARE). It allocates and uses periodic time slots for QoS-demanding applications. DARE reserves these time slots in a fully distributed way, schedules the realtime data packets, repairs broken reservations, and disseminates the reservation information to potential interferers using a piggyback technique. It works well for high traffic load network but poorly for low traffic load network. [4] presented a distributed MAC scheme called opportunistic synchronous array method (SAM) for a large network of wireless routers. [5] presented a novel tone-based contention resolution mechanism that exploits space-time uncertainty and high latency to detect collisions and count contenders, achieving good throughput across all offered loads for underwater acoustic sensor networks.

In this paper, an adaptive EDCA scheme with QoS guarantee for MANETs is proposed. In this scheme, the EDCA algorithm and the token bucket algorithm (TBA) are combined to adjust the $\mathrm{CW}$. Our scheme provides the traffic differentiation.

\section{Token Bucket Algorithm}

The number of bytes in the bucket (bucket length) and the occupancy of the transmission buffer (queue length) as input parameters are employed in the token bucket algorithm [6] (see Figure 1).

The bucket size (bsize) determines the accepted burstiness of source. The bucket length (blen) represents the resources that the user can use to transmit packets. The bucket limit (blim) represents the maximum packet length allowed. The queue size (qsize) represents the source capacity. The queue length (qlen) denotes the willingness of a station to transmit packets.

This algorithm computes a value which is used to scale the CW values defined in IEEE 802.11. It is described as follows:

If (overload) then $\mathrm{p}=(1+\Delta 4) \mathrm{p}$

Else if $(q$ len $=0)$ then $p=(1+\Delta 1) p$

Else if $($ blen $<$ blim $)$ then $p=(1+\Delta 2) p$

Else $\mathrm{p}=(1-\Delta 3) \mathrm{p}$

$\mathrm{P}=\min \{\mathrm{p}, 1\}$

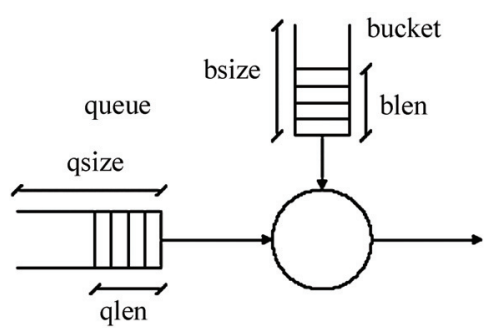

Figure 1. Brief description of token bucket algorithm.

\section{$\mathrm{CW}=\mathrm{p}^{*} \mathrm{CW} 802.11$}

where $\Delta 1=0.025, \quad \Delta 4=0.25, \quad \Delta_{2}=\frac{b \lim -b l e n}{b \lim } \Delta_{1}$ ， $\Delta_{3}=\frac{b l e n-b \lim }{b s i z e-b \lim } \Delta_{1}$. A solution to determine the overload is described as follows:

If (av_nr_coll $>$ c) then overload $=$ true

Av_nr_coll $=(1-\mathrm{t}) *$ num_coll $+\mathrm{av} \_n \mathrm{nr}$ coll

where av_nr_coll is the average collision number; $\mathrm{c}$ is a constant with value in $[0,8]$ and is always set to 4 ; $\mathrm{t}=0.25$; num coll is the collision number after transmission.

\section{Adaptive EDCA}

EDCA [7] is designed to provide prioritized QoS by enhancing the contention-based DCF. It provides differentiated, distributed access to the wireless medium for QoS stations (QSTAs) using 8 different user priorities (UPs). Before entering the MAC layer, each data packet received from the higher layer is assigned a specific user priority value. How to tag a priority value for each packet is an implementation issue. The EDCA scheme defines four different first-in first-out (FIFO) queues called access categories (ACs) that provide QoS support for the delivery of traffic with UPs at the QSTAs. Each data packet from the higher layer along with a specific user priority value is mapped into a corresponding AC according to Table 1. Different kinds of applications (e.g., background traffic, best effort traffic, video traffic, and voice traffic) can be casted into different ACs. For each $\mathrm{AC}$, an enhanced version of the DCF, called enhanced distributed channel access function (EDCAF), contends for TXOPs using a set of EDCA parameters from the EDCA Parameter Set Element or from the default values of the parameters when no EDCA Parameter Set Element is received from the QAP of the QBSS with which the QSTA is associated. [8] proposed an effective and simple model for two-level collision to demonstrate that EDCA

Table 1. User priority to access category mappings.

\begin{tabular}{ccc}
\hline $\begin{array}{c}\text { User priority } \\
\text { (UP) }\end{array}$ & Access category (AC) & Designation \\
\hline 1 & 0 & Background \\
2 & 0 & Background \\
0 & 1 & Best effort \\
3 & 1 & Best effort \\
4 & 2 & Video \\
5 & 2 & Video \\
6 & 3 & Voice \\
7 & 3 & Voice \\
\hline
\end{tabular}




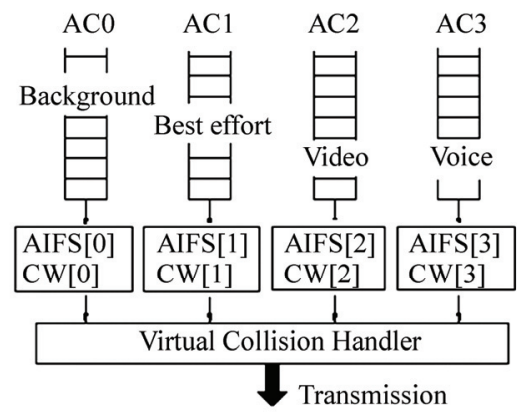

Figure 2. Implementation model.

mechanism, which adopts priority classification and two-level collision, can not only preferentially guarantee the throughput of voice and video, but also greatly decrease the average delay of the system.

Figure 2 shows the implementation model with four transmission queues, where each $\mathrm{AC}$ behaves like a virtual station: It contends for access to the medium and independently starts its backoff procedure after sensing the medium idle for at least AIFS period. In EDCA a new type of IFS is introduced, the arbitrary IFS (AIFS), in place of DIFS in DCF. Each AIFS is an IFS interval with arbitrary length as follows:

$$
\text { AIFS }[\mathrm{AC}]=\mathrm{SIFS}+\mathrm{AIFSN}[\mathrm{AC}] \times \text { slot time }
$$

where AIFSN[AC] is called the arbitration IFS number and is determined by the $\mathrm{AC}$ and the physical settings. The timing relationship of EDCA is shown in Figure 3. The AC with the smallest AIFS has the highest priority. The values of AIFS [AC], CWmin [AC], and CWmax $[\mathrm{AC}]$, which are referred to as the EDCA parameters, are announced by the AP via beacon frames. The purpose of using different contention parameters for different queues is to give a low-priority class a longer waiting time than a high-priority class, so the high-priority class is likely to access the medium earlier than the low-priority class. [9] presented the effect of different values of AIFS on MAC access delay. An internal collision occurs when more than one AC finishes the backoff procedure at the same time. In such a case, a virtual collision handler in every QSTA allows only the highest-priority AC to transmit frames, and the others per- form a backoff operation with increased $\mathrm{CW}$ values. [10] proposed an analytical model to calculate the EDCA parameter set that ideally achieves a predetermined utilization ratio between uplink and downlink flows.

Transmission opportunity (TXOP) is defined in IEEE 802.11e as the interval of time when a particular QSTA has the right to initiate transmissions. There are two modes of EDCA TXOP defined, the initiation of the EDCA TXOP and the multiple frame transmission within an EDCA TXOP. An initiation of the TXOP occurs when the EDCA rules permit access to the medium. A multiple frame transmission within the TXOP occurs when an EDCAF retains the right to access the medium following the completion of a frame exchange sequence, such as on receipt of an ACK frame. The TXOP limit duration values are advertised by the QAP in the EDCA Parameter Set Information Element in Beacon frames. During an EDCA TXOP, a STA is allowed to transmit multiple MAC protocol data units (MPDUs) from the same AC with a SIFS time gap between an ACK and the subsequent frame transmission. A TXOP limit value of 0 indicates that a single MPDU may be transmitted for each TXOP. This is also referred to as contention free burst (CFB).

We propose an adaptive EDCA scheme for the contention period (CP). In our scheme, we combine the EDCA scheme and the token bucket algorithm (TBA) to adjust the $\mathrm{CW}$. In the adjustment of the $\mathrm{CW}$, there are additional aspects that have to be taken into account: 1) We do not want the $\mathrm{CW}$ to increase above the values used by the Best Effort terminals, since this would lead to a worse performance than Best Effort. For backward compatibility, the $\mathrm{CW}$ for Best Effort should be the one defined by the 802.11 standard; 2) If the low transmission rate of the application is the reason for transmitting below the desired rate, then the CW should obviously not be decreased; 3) When estimating the transmission rate, it would be desirable to control the allowed burstiness of the source; 4) CWs should not be allowed to decrease in such a way that they negatively influence the overall performance of the network. After considering all the above issues, we describe the adaptive EDCA scheme as follows.

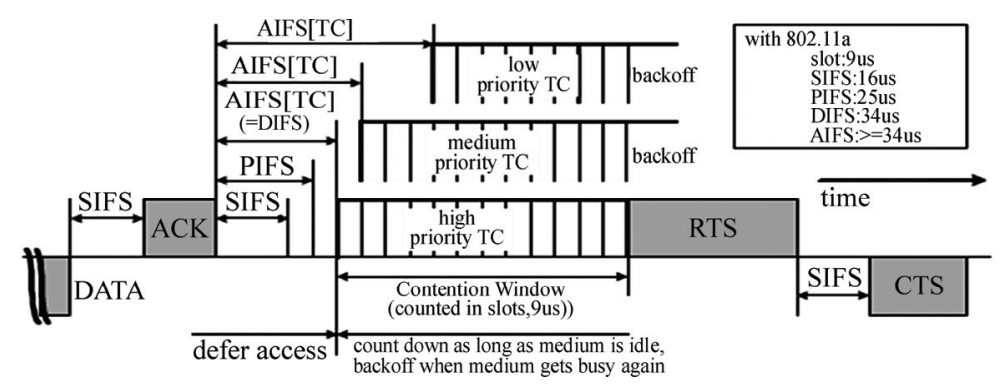

Figure 3. The timing relationship of EDCA. 


\subsection{Successful Transmission}

Step 1. Compute the collision rate

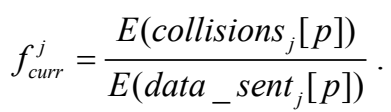

Step 2. Introduce exponentially weighted moving average (EWMA) to compute the average collision rate

$$
f_{\text {avg }}^{j}=(1-\alpha) * f_{\text {curr }}^{j}+\alpha^{*} f_{\text {avg }}^{j-1},
$$

where $\alpha$ is between 0.2 and 0.25 .

Step 3. Compute $\mathrm{p}$ by using the TBA

If $($ overload $)$ then $p=(1+\Delta 4) p$

Else if $(q$ len $=0)$ then $p=(1+\Delta 1) p$

Else if $($ blen $<$ blim $)$ then $p=(1+\Delta 2) p$

Else $p=(1-\Delta 3) p$

$\mathrm{P}=\min \{\mathrm{p}, 1\}$,

where $\Delta 1=0.025, \quad \Delta 4=0.25, \quad \Delta_{2}=\frac{b \lim -\text { blen }}{b \lim } \Delta_{1}$, $\Delta_{3}=\frac{b l e n-b \lim }{b s i z e-b \lim } \Delta_{1}$.

Step 4. Use the multiply factor (MF)

$\operatorname{MF}[i]=\min (\mathrm{p}, 0.8)$.

Step 5. After successful transmission, $\mathrm{CW}[\mathrm{i}]$ is set to CWnew $[\mathrm{i}]=\max (\mathrm{CWmin}[\mathrm{i}], \mathrm{CWold}[\mathrm{i}] * \mathrm{MF}[\mathrm{i}])$.

\subsection{After Collision}

Step 1. Compute the collision rate

$$
f_{\text {curr }}^{j}=\frac{E\left(\text { collisions }_{j}[p]\right)}{E\left(\text { data_sent }_{j}[p]\right)} .
$$

Step 2. Introduce exponentially weighted moving average (EWMA) to compute the average collision rate

$$
f_{\text {avg }}^{j}=(1-\alpha)^{*} f_{\text {curr }}^{j}+\alpha^{*} f_{\text {avg }}^{j-1},
$$

where $\alpha$ is between 0.2 and 0.25 .

Step 3. Compute $\mathrm{p}$ by using TBA

If (overload) then $\mathrm{p}=(1+\Delta 4) \mathrm{p}$

Else if $(q$ len $=0)$ then $p=(1+\Delta 1) p$

Else if $($ blen $<$ blim $)$ then $p=(1+\Delta 2) p$

Else $p=(1-\Delta 3) p$

$\mathrm{P}=\min \{\mathrm{p}, 1\}$

If (overload) then $\mathrm{p}=(1+\Delta 4) \mathrm{p}$

If $\left(f_{\text {avg }}^{j}>c\right)$ then overload=true,

where $\mathrm{c}=0.5, \Delta 1=0.025, \Delta 4=0.25, \Delta_{2}=\frac{b \lim -\text { blen }}{b \lim } \Delta_{1}$, $\Delta_{3}=\frac{b l e n-b \lim }{b s i z e-b \lim } \Delta_{1}$.

Step 4. Use the multiply factor (MF)

$$
\operatorname{MF}[\mathrm{i}]=\min (\mathrm{p}, 0.8) \text {. }
$$

Step 5. After transmission failure, $\mathrm{CW}[\mathrm{i}]$ is set according to each TC's $\mathrm{PF}[\mathrm{i}]$ to guarantee that high priority TC has low PF[i] value

CWtemp[i] $=\min (\mathrm{CWmax}[\mathrm{i}], \mathrm{CWold}[\mathrm{i}] * \mathrm{MF}[\mathrm{i}])$

CWnew $[\mathrm{i}]=\max \left(\mathrm{CW}\right.$ old $[\mathrm{i}], \mathrm{CW}$ temp $\left.[\mathrm{i}]^{*} \mathrm{MF}[\mathrm{i}]\right)$.

\section{Simulation Results}

In this section we evaluate the performance of EDCF (IEEE 802.11e) [11], AEDCF and our scheme (Adaptive EDCA). AEDCF [12] is another improved scheme of EDCA. It also improves the throughput through changing $\mathrm{CW}$.

The simulation is with respect to two scenarios, namely Scenario 1 and Scenario 2. Scenario 1 is a light traffic load environment. Scenario 2 is a high traffic load environment. The parameters CWmin, CWmax, AIFS are set to $5,100,5$ in the scenario 1 and $30,500,15$ in the scenario 2 . In our scheme $p, \alpha$, bsize, and $\mathrm{c}$ are set to $0.5,0.25,10,0.5$, respectively. Through Figure 4 we find that there is a slump of EDCF and AEDCF in the 10 stations because of collision among these stations. But our scheme (Adaptive EDCA) keep good throughput by using TBA.

Through Figure 5 we find that the throughput of AEDCF is $4.9 \%$ higher than EDCF in the high traffic load environment. But the throughput of our scheme (Adaptive EDCA) is $11.4 \%$ higher than EDCA.

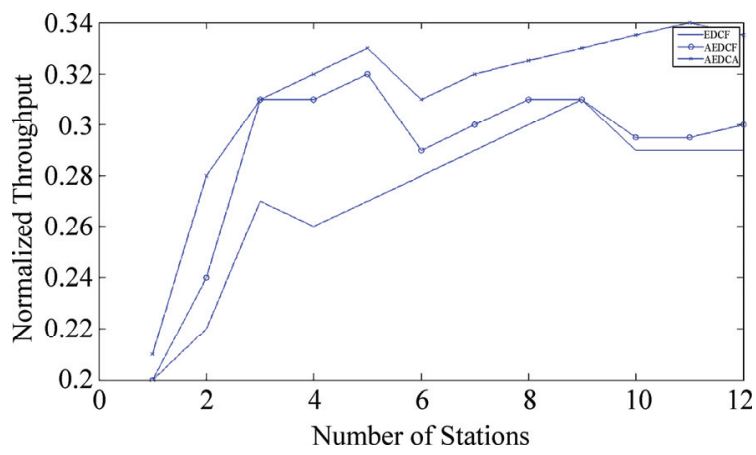

Figure 4. Scenario 1.

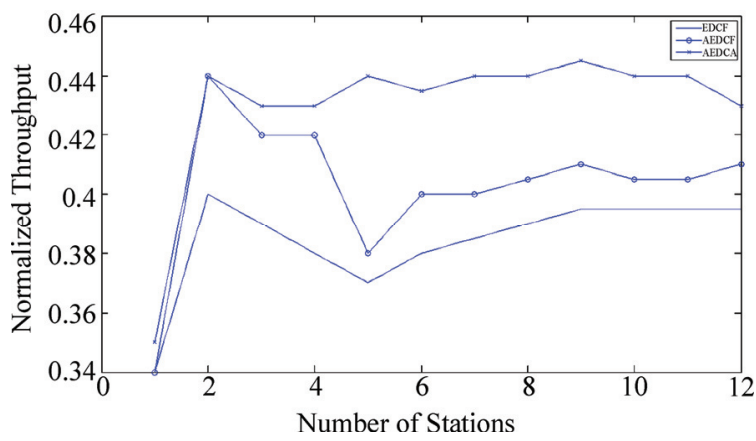

Figure 5. Scenario 2. 


\section{Conclusions}

The real-time and multimedia services are more and more popular. We find that providing service differentiation and high throughput is a new challenge in MANETs. The MAC protocol of the IEEE 802.11e is changed in order to improve its effectiveness. The EDCA scheme and the TBA are combined to adjust the $\mathrm{CW}$. Our scheme provides the traffic differentiation. The results show the good performance of our scheme under both light and high traffic loads.

\section{References}

[1] IEEE Std, “802.11-1999, Part 11: Wireless LAN Medium Access Control (MAC) and Physical Layer (PHY) Specifications," Reference number ISO/IEC 8802-11:1999(E), IEEE Std. 802.11, 1999 Edition.

[2] P. V. Krishna and Iyengar, "Sequencing technique: An enhancement to 802.11 medium access control to improve the performance of wireless networks," N.Ch.S.N., International Journal Communication Networks and Distributed Systems, Vol. 1, No. 1, pp. 52-70, 2008.

[3] E. Carlson, C. Prehofer, C. Bettstetter, et al, "A distributed end-to-end reservation protocol for IEEE 802.11based wireless mesh networks," IEEE Journal on Selected Areas in Communications, Vol. 24, No. 11, November 2006, pp. 2018-2027.

[4] B. Zhao and Y. B. Hua, "A distributed medium access control scheme for a large network of wireless routers," IEEE Transactions on Wireless Communications, Vol. 7, No. 5, pp. 1614-1622, May 2008.

[5] A. A. Syed, W. Ye, and J. Heidemann, "T-Lohi: A new class of MAC protocols for underwater acoustic sensor networks," IEEE INFOCOM'08, The 27th Conference on Computer Communications, pp. 231-235, 13-18 April 2008.

[6] A. Banchs and X. Perez, "Providing throughput guarantees in IEEE 802.11 wireless LAN," Wireless Communications and Networking Conference, IEEE WCNC'02, Vol. 1, pp. 130-138, 17-21 March 2002.

[7] S. Sehrawat, R. P. Bora, and D. Harihar, "Performance analysis of QoS supported by enhanced distributed channel access (EDCA) mechanism in IEEE 802.11e," IAENG International Journal of Computer Science, IJCS_33_1_6, Vol. 33, No. 1, February 2007.

[8] J. R. Yan, S. Y. Zhang, H. Long, and Y. F. Sun, "An analytical model for EDCA mechanism," Journal of Electronics \& Information Technology, Vol. 30, No. 4, April 2008 .

[9] X. Y. Zhou, H. F. W, L. M. Sun, et al., "MAC access delay analysis of EDCA mechanism of wireless LANs," Journal of Software, Vol. 19, No. 8, pp. 2127-2139, 2008. http://www.jos.org.cn/1000-9825/19/2127.htm.

[10] F. Keceli, I. Inan, and E. Ayanoglu, "Weighted fair uplink/downlink access provisioning in IEEE 802.11e WLANs," ICC'08, IEEE International Conference on Communications, pp. 2473-2479, 19-23 May 2008.

[11] S. H. Choi, J. del Prado, N. S. Shankar, et al., "IEEE 802.11 e contention-based channel access (EDCF) performance evaluation," ICC'03, IEEE International Conference on Communications, pp. 1151-1156, 11-14 May 2003.

[12] L. Romdhani, Q. Ni, T. Turletti, "Adaptive EDCF: Enhanced service differentiation for IEEE 802.11 wireless ad-hoc networks," IEEE Wireless Communications and Networking, Conference, Vol. 2, pp. 16-20, March 2003. 